\title{
Improvement in Patient-Reported Outcomes (Dermatology Life Quality Index and the Psoriasis Symptoms and Signs Diary) with Guselkumab in Moderate-to-Severe Plaque Psoriasis: Results from the Phase III VOYAGE 1 and VOYAGE 2 Studies
}

\author{
April W. Armstrong ${ }^{1} \cdot \mathrm{Kristian}$ Reich $^{2,3} \cdot$ Peter Foley $^{4} \cdot$ Chenglong Han $^{5} \cdot$ Michael Song $^{5} \cdot$ Yaung-Kaung Shen ${ }^{5}$. \\ Yin You ${ }^{5} \cdot$ Kim A. Papp ${ }^{6}$
}

Published online: 12 November 2018

(c) The Author(s) 2018

\begin{abstract}
Background Health-related quality of life (HRQoL) may be markedly impaired in patients with moderate-to-severe psoriasis. Objectives Our objectives were to compare improvements in Dermatology Life Quality Index (DLQI) and Psoriasis Symptoms and Signs Diary (PSSD) scores between patients receiving guselkumab compared with placebo or adalimumab and to correlate these improvements with skin clearance.

Methods Pooled phase III VOYAGE 1 and VOYAGE 2 data were evaluated through week 24. At baseline, patients were randomized to guselkumab $100 \mathrm{mg}$, placebo, or adalimumab $40 \mathrm{mg}$. At week 16, patients receiving placebo switched to guselkumab. Assessment measures included DLQI percent change from baseline, DLQI 0/1, DLQI minimal clinically important difference (MCID), individual domain scores, PSSD symptoms and signs score =0, DLQI association with PSSD, Investigator's Global Assessment (IGA), and Psoriasis Area and Severity Index (PASI)

Results Significantly greater improvements from baseline DLQI were observed with guselkumab versus placebo (weeks 8 and 16) and versus adalimumab (week $24 ; p<0.001$ ). The proportion of patients achieving DLQI 0/1 ("no impact") at week 24 was higher with guselkumab than with adalimumab (58.9 vs. $40.2 \% ; p<0.001$ ), and more patients attained a $\geq 4$-point reduction in DLQI (MCID) at this timepoint $(p<0.001)$. Changes in individual DLQI domains were significantly greater for patients receiving guselkumab than for those receiving adalimumab, and among patients with individual baseline domain scores $=3$ or 6 (severest impact), more guselkumab recipients than those receiving adalimumab achieved a score $=0$ across all domains at week 24. DLQI 0/1 scores were associated with a PSSD symptom or sign score $=0$ (no impact) and greater improvement of PASI and IGA (week 24).

Conclusions Pooled VOYAGE 1/VOYAGE 2 data demonstrated that guselkumab was superior to adalimumab in improving HRQoL, which was associated with greater skin clearance.
\end{abstract}

Clinical Trial Registration NCT02207231 and NCT02207244.

April W. Armstrong

aprilarmstrong@post.harvard.edu

1 Keck School of Medicine of USC, University of Southern California, 1975 Zonal Avenue, Keith Administration Building, Room 510, Los Angeles, CA 90089, USA

2 Dermatologikum Berlin, Berlin, Germany

3 SCIderm Research Institute, Hamburg, Germany

4 The University of Melbourne, St. Vincent's Hospital Melbourne, Fitzroy and Probity Medical Research Inc., and Skin \& Cancer Foundation Inc., Carlton, VIC, Australia
5 Janssen Research \& Development, LLC, Spring House, PA, USA

6 K Papp Clinical Research and Probity Medical Research Inc., Waterloo, ON, Canada 


\section{Key Points}

Health-related quality of life (HRQoL) can be severely impaired in patients with moderate-to-severe psoriasis.

Our study showed that guselkumab was superior to adalimumab in improving HRQoL. Additionally, greater improvement in psoriasis severity was associated with greater improvement in HRQoL.

These findings support the relevance of the patient's perception of the impact of psoriasis on HRQoL and further validates the increasing emphasis on patient-reported outcomes in evaluating clinical responses to treatment.

\section{Introduction}

Psoriasis is a chronic disease with predominant skin signs and symptoms of erythema, scaling, pruritus, and pain, resulting in psychological impact and reduced quality of life [1-3]. The importance of patient-reported assessments of improvement in health-related quality of life (HRQoL) has been recognized. The Dermatology Life Quality Index (DLQI) was developed to measure a patient's perception of the effect of skin disease on his/her daily life [4]. A DLQI goal of 0 or 1, indicating no impact on quality of life, is a standard measure in clinical studies for skin diseases [5]. Additionally, the minimal clinically important difference (MCID) for the DLQI, the smallest score difference that patients perceive as beneficial [6], has varied, having been previously defined as 6 [7] or 5 [7, 8] but most recently clarified as 4 [9].

Increasingly, greater emphasis has been placed on HRQoL in clinical studies, and multiple new tools to assess HRQOL have been created and validated [10-12]. The Psoriasis Symptoms and Signs Diary (PSSD) [10, 13] is a validated tool that measures psoriasis-specific symptoms and signs and disease severity and was used to evaluate these parameters in the guselkumab psoriasis program. Consistent with the negative impact of psoriasis, patients often desire clear skin $[14,15]$. Treatment with biologics that block the interleukin (IL)-23/IL-17 immune pathway has been shown to result in clear or almost clear skin in the majority of patients with psoriasis [16-22]. Data are conflicting as to whether greater improvements in Psoriasis Area and Severity Index (PASI) scores correlate with lower DLQI scores [23-29], which may be partly due to the methodology of the analyses. Therefore, it is important that new psoriasis treatments should not only improve a patient's skin clearance as assessed by the physician but also demonstrate improvement from the patient's perspective, utilizing newer assessment tools, in addition to the DLQI, that focus on patient-reported evaluations of the symptoms and signs of psoriasis [10, 13].
Guselkumab (TREMFYA ${ }^{\circledR}$; Janssen Research \& Development LLC, Spring House, PA, USA) is a fully human monoclonal antibody that binds to the p19 subunit of IL-23 and inhibits the intracellular and downstream signaling of IL-23. Results for efficacy, safety, and patient-reported outcomes of guselkumab compared with adalimumab were previously reported for two pivotal, phase III clinical trials (VOYAGE 1 and VOYAGE 2) [16, 17]. Here, we present the pooled DLQI and PSSD data from VOYAGE 1 and VOYAGE 2 and evaluate the associations between DLQI and improvements in PSSD, PASI, and Investigator's Global Assessment (IGA).

\section{Patients and Methods}

\subsection{Study Design}

VOYAGE 1 (NCT02207231) and VOYAGE 2 (NCT02207244) are phase III, multicenter, randomized, double-blinded, placebo- and adalimumab comparatorcontrolled studies with identical designs through week 24. Patients were randomized at baseline to guselkumab $100 \mathrm{mg}$ at weeks $0,4,12$, and 20; placebo at weeks 0,4 , and 12 followed by guselkumab $100 \mathrm{mg}$ at weeks 16 and 20; or adalimumab $80 \mathrm{mg}$ at week 0 , adalimumab $40 \mathrm{mg}$ at week 1 , and adalimumab $40 \mathrm{mg}$ every 2 weeks through week 24 . In both studies, patients were $>18$ years old had a diagnosis of plaque-type psoriasis for $\geq 6$ months before the first administration of study agent, had a baseline PASI $\geq 12$, IGA score $\geq 3$, an involved body surface area of $\geq 10 \%$, and were a candidate for phototherapy/systemic psoriasis treatments. Exclusion criteria and other details of the studies have been reported previously $[16,17]$. Informed consent was obtained from all individual participants included in these studies. The analyses in this report were performed using the pooled data from the VOYAGE 1 [16] and VOYAGE 2 [17] studies.

\subsection{Assessments}

Skin-related HRQoL was assessed using the DLQI and PSSD measures. The DLQI has ten questions related to the effect of skin problems on six aspects of life-labeled individual domains: symptoms and feelings (two questions), daily activities (two questions), leisure (two questions), work and school performance (one question), personal relationships (two questions), and treatment (one question). Each question is scored from 0 (not at all) to 3 (very much), indicating the intensity of impact on individual aspects of life. The total domain score varies from 0 to 3 in domains with one question and from 0 to 6 in domains with two questions. For example, a score of 0 indicates no impact at all of psoriasis on an individual functional domain, and a score of 3 or 6 (depending on the number of questions) indicates the most severe impact. 
The overall DLQI score is the sum of the individual questions and ranges from 0 (meaning no impact of skin disease on HRQoL) to 30 (meaning maximum impact on HRQoL) [4]. Overall DLQI measures included percent change from baseline, a score of 0 or 1 (no impact of psoriasis on a patient's HRQoL), and the MCID (the smallest score difference that patients perceive as beneficial), evaluated as a change in score of 4,5 , or 6 points.

The PSSD evaluates symptoms (i.e., itch, pain, stinging, burning, and skin tightness) and signs (skin dryness, cracking, scaling, shedding or flaking, redness, and bleeding) of psoriasis, graded individually ( $0-10$ scale) by the patient using electronic capture in a daily diary requiring $24-\mathrm{h}$ recall. A higher score indicates more severe symptoms or signs of psoriasis $[10,13]$. The proportions of patients achieving PSSD symptom and sign summary scores of 0 (i.e., free of symptoms and signs) at week 24 were reported.

Clinical disease severity was evaluated by clinicians using two instruments: the IGA (range 0 [cleared] to 4 [severe]) and PASI (range 0-72; a higher score indicates more severe disease) [30]. Pooled data from both studies through week 24 were used to evaluate DLQI improvement and its relationship to PSSD and objectively measured skin improvement.

\subsection{Statistical Analyses}

DLQI scores were analyzed in patients with a DLQI score evaluated at baseline. Endpoints included the proportion of patients achieving a DLQI score of 0/1 with DLQI baseline score $>1$, as well as the change in DLQI score from baseline to weeks 8,16 , and 24 . Additionally, the percent improvement in the individual domains and the proportion of patients achieving an individual domain score of 0 (no impact) were assessed among patients who had corresponding scores of 3 (most severe impact) at baseline. Binary endpoints were analyzed using a Cochran-Mantel-Haenszel Chi squared test stratified by study and investigator site, and continuous endpoints were analyzed using an analysis of variance model with study and investigator site as covariates. After treatment failure rules were applied, nonresponder imputation rules were applied for binary endpoints, and last observation carried forward rules were applied for continuous variables, respectively, for the remaining missing data. Nominal $p$-values were reported for these post hoc analyses.

\section{Results}

\subsection{Baseline Demographics and Disease Characteristics}

A total of 1829 patients were analyzed in the pooled VOYAGE 1 [16] and VOYAGE 2 [17] studies: VOYAGE 1 included 837 patients (guselkumab 329; adalimumab 334; placebo 174), and VOYAGE 2 included 992 patients (guselkumab 496; adalimumab 248; placebo 248). Baseline demographics and psoriasis disease characteristics were comparable across the treatment groups of the pooled population (Table 1) and were consistent with those for the treatment groups in the individual studies. The median DLQI score at baseline was 14.0 in each group.

\subsection{Efficacy}

\subsubsection{Changes in Dermatology Life Quality Index (DLQI) Scores from Baseline}

A significantly greater reduction (improvement) in DLQI score from baseline was observed in guselkumab-treated patients $(-9.6 \pm 6.7)$ compared with placebo-treated patients $(-1.7 \pm 5.9)(p<0.001)$ at week 8 , the first timepoint of DLQI evaluation after baseline, and at week $16(-11.3 \pm 7.0$ vs. $-1.8 \pm 6.7$, respectively; $p<0.001$ ) (Table 2 ).

At week 24, significant improvements were observed in the guselkumab group compared with the adalimumab group ( $-11.8 \pm 7.2$ vs. $-9.6 \pm 7.7$, respectively; $p<0.001$; Table 2). At week 24, improvements in DLQI were similar between the placebo-crossover patients and those originally randomized to guselkumab (data not shown).

Among the patients with a DLQI score $>1$ at baseline, significantly greater proportions of guselkumab-treated patients achieved a DLQI score of $0 / 1$ at weeks 8 and 16 compared with placebo-treated patients $(p<0.001$ for all; Fig. 1). Likewise, a significantly greater proportion of guselkumab-treated patients achieved a DLQI score of $0 / 1$ compared with adalimumab-treated patients at week 24 ( $p<0.001$; Fig. 1).

In addition, significantly greater proportions of guselkumab-treated patients achieved $\mathrm{a} \geq 4$-point (88.7 vs. $79.0 \%$ ), $\geq 5$-point ( 88.4 vs. $76.3 \%$ ), or $\geq 6$-point (87.3 vs. $75.2 \%$ ) reduction in DLQI score at week 24 compared with adalimumab-treated patients ( $p<0.001$ for all; Fig. 2).

At week 24, changes in each of the individual DLQI domains were significantly greater for guselkumab-treated patients than for adalimumab-treated patients $(p<0.001)$. The greatest numerical difference in percent improvement was observed for the "Symptoms and Feelings" domain ( $74.5 \pm 31.8$ vs. $57.4 \pm 47.9$, respectively; Table 3 ).

At baseline, a substantial proportion of patients in the guselkumab and adalimumab groups experienced a severe impact (score of 3 [one question] or score of 6 [two questions]) of psoriasis on HRQoL. Following treatment, among patients with a baseline average domain score of 3 or 6 (depending on the question), across all domains, greater proportions of guselkumab-treated patients achieved a score of 0 (no impact) 
Table 1 Baseline disease characteristics

\begin{tabular}{|c|c|c|c|c|}
\hline & Placebo & Guselkumab & Adalimumab & Total \\
\hline Patients randomized at week $0, N$ & 422 & 825 & 582 & 1829 \\
\hline \multicolumn{5}{|l|}{ Psoriasis disease duration, years } \\
\hline$N$ & 422 & 825 & 582 & 1829 \\
\hline Mean \pm SD & $17.8 \pm 12.1$ & $17.9 \pm 12.1$ & $17.3 \pm 11.4$ & $17.7 \pm 11.9$ \\
\hline Median & 15.0 & 16.0 & 15.0 & 15.0 \\
\hline \multicolumn{5}{|l|}{ BSA, \% involvement } \\
\hline$N$ & 422 & 825 & 582 & 1829 \\
\hline Mean \pm SD & $27.1 \pm 16.3$ & $28.4 \pm 16.7$ & $28.8 \pm 16.7$ & $28.2 \pm 16.6$ \\
\hline Median & 21.0 & 23.0 & 24.0 & 23.0 \\
\hline \multicolumn{5}{|l|}{ DLQI score (0-30) } \\
\hline$N$ & 418 & 817 & 575 & 1810 \\
\hline Mean \pm SD & $14.3 \pm 7.2$ & $14.4 \pm 7.2$ & $14.6 \pm 7.1$ & $14.5 \pm 7.2$ \\
\hline Median & 14.0 & 14.0 & 14.0 & 14.0 \\
\hline \multicolumn{5}{|l|}{ PASI score $(0-72)$} \\
\hline$N$ & 422 & 825 & 582 & 1829 \\
\hline Mean \pm SD & $21.1 \pm 8.3$ & $22.0 \pm 9.1$ & $22.1 \pm 9.0$ & $21.8 \pm 8.9$ \\
\hline Median & 18.3 & 19.0 & 19.5 & 19.0 \\
\hline \multicolumn{5}{|l|}{ IGA score $(0-4), N(\%)$} \\
\hline$N$ & 422 & 825 & 582 & 1829 \\
\hline Moderate (3) & $322(76.3)$ & $632(76.6)$ & $436(74.9)$ & $1390(76.0)$ \\
\hline Severe (4) & $100(23.7)$ & $192(23.3)$ & $143(24.6)$ & $435(23.8)$ \\
\hline \multicolumn{5}{|l|}{ PSSD symptom score $(0-100)$} \\
\hline$N$ & 327 & 660 & 475 & 1462 \\
\hline Mean \pm SD & $54.5 \pm 24.1$ & $54.2 \pm 25.6$ & $53.8 \pm 25.9$ & $54.2 \pm 25.3$ \\
\hline Median & 54.0 & 54.0 & 56.0 & 54.0 \\
\hline \multicolumn{5}{|l|}{ PSSD sign score (0-100) } \\
\hline$N$ & 327 & 660 & 475 & 1462 \\
\hline Mean \pm SD & $58.1 \pm 20.5$ & $56.5 \pm 22.1$ & $57.8 \pm 21.6$ & $57.3 \pm 21.6$ \\
\hline Median & 58.0 & 57.5 & 58.0 & 58.0 \\
\hline
\end{tabular}

BSA body surface area, DLQI Dermatology Life Quality Index, IGA Investigator's Global Assessment, PASI Psoriasis Area and Severity Index, PSSD Patient Symptoms and Signs Diary, $S D$ standard deviation

at week 24 compared with adalimumab-treated patients (Table 4). Overall, in the pooled analysis of all patients at week 24, change from baseline in PASI score was significantly correlated with change in DLQI total score $(r=0.375 ; p<0.001$; data not shown).

\subsubsection{Changes in Psoriasis Symptoms and Signs Diary (PSSD) Scores from Baseline}

A significantly greater proportion of patients achieved a PSSD symptom score of 0 at week 24 with guselkumab versus adalimumab treatment ( 35.6 vs. $22.0 \%$; $p<0.001$ ). Similar results were observed for the proportion of patients achieving a PSSD sign score of 0 at week 24 (guselkumab: $28.4 \%$ vs. adalimumab: $15.6 \% ; p<0.001)$. Overall, in the pooled analysis of all patients at week 24 , changes from baseline in PASI score significantly correlated with changes in PSSD symptom score $(r=0.307 ; p<0.001)$ and changes in PSSD sign score $(r=0.301 ; p<0.001)$ (data not shown).

\subsubsection{DLQI 0/1 Response by Psoriasis Area and Severity Index (PASI), Investigator's Global Assessment (IGA), and PSSD Responses}

Stratified by the improvement in PASI, IGA, and PSSD scores, our analysis showed a clear trend indicating that greater improvement in psoriasis severity was associated with greater improvement in DLQI 0/1. Additionally, regardless of the level of clinical response assessed by PASI or IGA, there was a trend showing that a greater proportion of guselkumab-treated patients achieved a DLQI score of 0/1 compared with adalimumab-treated patients (Table 5). 
Table 2 Change in Dermatology Life Quality Index scores from baseline through week 24

\begin{tabular}{llll}
\hline & Placebo & Guselkumab & Adalimumab \\
\hline $\begin{array}{l}\text { Patients randomized at } \\
\text { week } 0, N\end{array}$ & 422 & 825 & 582 \\
Week 8 & & & \\
$N$ & & & 575 \\
Mean \pm SD & 418 & 817 & $-8.8 \pm 6.8$ \\
Median & $-1.7 \pm 5.9$ & $-9.6 \pm 6.7$ & -8.0 \\
$p$ value vs. placebo & -2.0 & -9.0 & $<0.001$ \\
Week 16 & & $<0.001$ & \\
$N$ & 418 & 817 & 575 \\
Mean \pm SD & $-1.8 \pm 6.7$ & $-11.3 \pm 7.0$ & $-9.5 \pm 7.4$ \\
Median & -1.0 & -11.0 & -9.0 \\
$p$ value vs. placebo & & $<0.001$ & $<0.001$ \\
Week 24 & & & \\
$N$ & NA & 817 & 575 \\
Mean \pm SD & NA & $-11.8 \pm 7.2$ & $-9.6 \pm 7.7$ \\
Median & NA & -12.0 & -9.0 \\
$p$ value vs. adalimumab & & $<0.001$ & \\
\hline
\end{tabular}

NA not applicable, $S D$ standard deviation

\section{Discussion}

In pooled data from the two pivotal phase III VOYAGE 1 [16] and VOYAGE 2 [17] studies, guselkumab was superior to adalimumab in improving HRQoL, which was assessed across multiple endpoints of the DLQI and PSSD measures. At week 24, patients treated with guselkumab had a significantly greater improvement in DLQI score from baseline than patients treated with adalimumab. In addition, a significantly greater proportion of guselkumab-treated patients achieved a DLQI $0 / 1$ and an MCID of 4,5 , or 6 compared with adalimumab-treated patients. Changes in each of the individual DLQI domains were significantly greater among guselkumab-treated patients than among adalimumab-treated patients at week 24 . A greater proportion of patients receiving guselkumab versus adalimumab achieved a PSSD score of 0 for both symptoms and signs at week 24. Improvements in HRQoL, defined as a DLQI 0/1, correlated with skin improvement, measured by both PASI and IGA. Achieving a DLQI of $0 / 1$ also correlated with achieving PSSD symptoms and signs scores of 0 . The totality of the data confirmed that, from both the physician and the patient perspective, guselkumab will be an effective

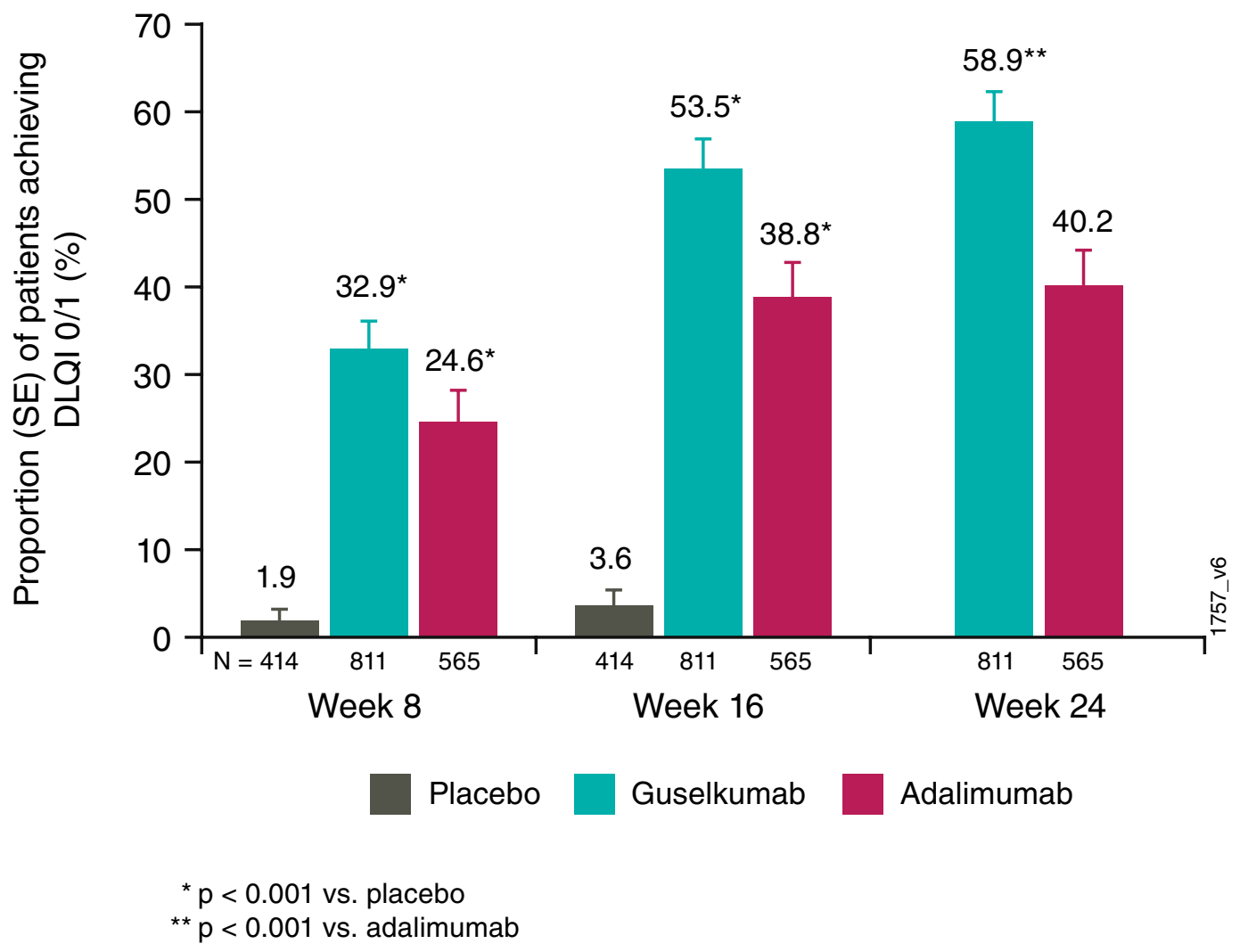

Fig. 1 Proportion of patients achieving a Dermatology Life Quality Index (DLQI) score of 0/1 at weeks 8, 16, and 24 in patients with baseline DLQI score $>1$. SE standard error 


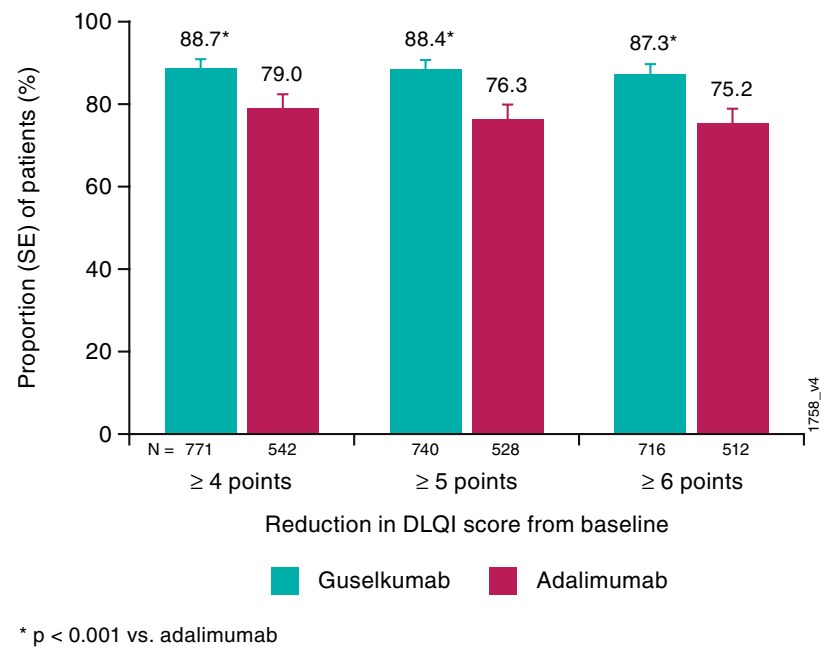

Fig. 2 Proportion of patients with a reduction of $4 / 5 / 6$ or more points in Dermatology Life Quality Index (DLQI) score from baseline to week 24 in patients with baseline DLQI scores of $\geq 4,5$, or 6 . $S E$ standard error

treatment for patients with moderate-to-severe psoriasis, particularly those desiring a high degree of efficacy.

Clinically meaningful improvement in the DLQI, a validated, 10-item, dermatology patient-reported outcome [9, $23,24]$, is defined as the smallest difference in score that a patient perceives as beneficial, commonly accepted to be 4 [9]. Failure to achieve this level of response would suggest, in the absence of adverse events or other precipitating factors, a need for change in the patient's management $[6$, 31]. Using this criterion and two higher MCID cutoffs of 5 and 6, guselkumab was superior to adalimumab. Further, using the most rigorous assessment, a significantly greater proportion of guselkumab-treated than adalimumab-treated patients achieved DLQI 0/1 at week 24. For each of the individual domains, the percent improvement was significantly greater in the guselkumab group than in the adalimumab group. Additionally, among patients who were most severely affected in each domain (baseline scores of 3 or 6), a greater number of guselkumab-treated patients than adalimumabtreated patients achieved a score of 0 across all six domains. This is particularly notable because comparative patientreported outcomes are increasingly recognized as important in psoriasis. This study demonstrated, using two different instruments, that patients assess guselkumab as superior to adalimumab, one of the most widely used biologics to treat psoriasis, in improving all aspects of their HRQoL. Other biologics targeting the IL-23 pathway, specifically IL-17, have also demonstrated superiority in improving HRQoL using the DLQI compared with established agents such as etanercept and ustekinumab [19-21, 32-34].

High levels of physician-assessed improvements in psoriasis in these studies, as assessed by the PASI and IGA
Table 3 Summary of change in Dermatology Life Quality Index (DLQI) domain scores from baseline to week 24

\begin{tabular}{|c|c|c|}
\hline & Guselkumab & Adalimumab \\
\hline Patients randomized at week $0, N$ & 825 & 582 \\
\hline \multicolumn{3}{|l|}{ DLQI domain scores } \\
\hline \multicolumn{3}{|l|}{ Symptoms and feelings } \\
\hline$N$ & 817 & 575 \\
\hline Mean change $\pm \mathrm{SD}$ & $-3.1 \pm 1.7$ & $-2.4 \pm 1.8$ \\
\hline Median change & -3.0 & -2.0 \\
\hline$p$ value vs. adalimumab & $<0.001$ & \\
\hline$N$ & 813 & 570 \\
\hline Mean $\%$ improvement $\pm \mathrm{SD}$ & $74.5 \pm 31.8$ & $57.4 \pm 47.9$ \\
\hline Median \% improvement & 80.0 & 66.7 \\
\hline$p$ value vs. adalimumab & $<0.001$ & \\
\hline \multicolumn{3}{|l|}{ Daily activities } \\
\hline$N$ & 817 & 575 \\
\hline Mean change \pm SD & $-2.6 \pm 1.8$ & $-2.1 \pm 1.8$ \\
\hline Median change & -3.0 & -2.0 \\
\hline$p$ value vs. adalimumab & $<0.001$ & \\
\hline$N$ & 751 & 530 \\
\hline Mean $\%$ improvement \pm SD & $84.0 \pm 34.8$ & $66.4 \pm 52.4$ \\
\hline Median \% improvement & 100.0 & 100.0 \\
\hline$p$ value vs. adalimumab & $<0.001$ & \\
\hline \multicolumn{3}{|l|}{ Leisure } \\
\hline$N$ & 817 & 575 \\
\hline Mean change $\pm S D$ & $-2.2 \pm 1.9$ & $-1.9 \pm 2.0$ \\
\hline Median change & -2.0 & -2.0 \\
\hline$p$ value vs. adalimumab & 0.017 & \\
\hline$N$ & 688 & 487 \\
\hline Mean $\%$ improvement \pm SD & $83.6 \pm 37.9$ & $69.3 \pm 56.0$ \\
\hline Median \% improvement & 100.0 & 100.0 \\
\hline$p$ value vs. adalimumab & $<0.001$ & \\
\hline \multicolumn{3}{|l|}{ Work and school } \\
\hline$N$ & 817 & 575 \\
\hline Mean change \pm SD & $-1.0 \pm 1.2$ & $-0.8 \pm 1.2$ \\
\hline Median change & -1.0 & -1.0 \\
\hline$p$ value vs. adalimumab & 0.016 & \\
\hline$N$ & 520 & 365 \\
\hline Mean $\%$ improvement $\pm \mathrm{SD}$ & $87.2 \pm 40.4$ & $72.9 \pm 51.2$ \\
\hline Median \% improvement & 100.0 & 100.0 \\
\hline$p$ value vs. adalimumab & $<0.001$ & \\
\hline \multicolumn{3}{|l|}{ Personal relationships } \\
\hline$N$ & 817 & 575 \\
\hline Mean change $\pm \mathrm{SD}$ & $-1.8 \pm 1.9$ & $-1.4 \pm 1.8$ \\
\hline Median change & -1.0 & -1.0 \\
\hline$p$ value vs. adalimumab & 0.001 & \\
\hline$N$ & 587 & 427 \\
\hline Mean $\%$ improvement \pm SD & $84.2 \pm 37.2$ & $66.4 \pm 63.2$ \\
\hline Median \% improvement & 100.0 & 100.0 \\
\hline$p$ value vs. adalimumab & $<0.001$ & \\
\hline
\end{tabular}


Table 3 (continued)

\begin{tabular}{lll}
\hline & Guselkumab & Adalimumab \\
\hline Treatment & & \\
$N$ & 817 & 575 \\
Mean change \pm SD & $-1.1 \pm 1.0$ & $-0.9 \pm 1.1$ \\
Median change & -1.0 & -1.0 \\
$p$ value vs. adalimumab & $<0.001$ & \\
$N$ & 607 & 423 \\
Mean \% improvement \pm SD & $86.2 \pm 33.5$ & $70.7 \pm 46.6$ \\
Median \% improvement & 100.0 & 100.0 \\
$p$ value vs. adalimumab & $<0.001$ & \\
\hline
\end{tabular}

$S D$ standard deviation

Table 4 Proportion of patients achieving scores of 0 at week 24 among patients with baseline Dermatology Life Quality Index (DLQI) individual domain scores of 3 or 6 (severe)

\begin{tabular}{|c|c|c|}
\hline & Guselkumab & Adalimumab \\
\hline Patients randomized at week $0, N$ & 825 & 582 \\
\hline \multicolumn{3}{|l|}{ Individual DLQI domain scores } \\
\hline \multicolumn{3}{|l|}{ Symptoms and feelings } \\
\hline Patients with baseline score $=6, N$ & 171 & 111 \\
\hline Patients with score $=0$ at week 24 & $67(39.2)$ & $20(18.0)$ \\
\hline$p$ value vs. adalimumab & $<0.001$ & \\
\hline \multicolumn{3}{|l|}{ Daily activities } \\
\hline Patients with baseline score $=6, N$ & 78 & 58 \\
\hline Patients with score $=0$ at week 24 & $45(57.7)$ & $16(27.6)$ \\
\hline$p$ value vs. adalimumab & 0.053 & \\
\hline \multicolumn{3}{|l|}{ Leisure } \\
\hline Patients with baseline score $=6, N$ & 89 & 70 \\
\hline Patients with score $=0$ at week 24 & $48(53.9)$ & $31(44.3)$ \\
\hline$p$ value vs. adalimumab & 0.501 & \\
\hline \multicolumn{3}{|l|}{ Work and school } \\
\hline Patients with baseline score $=3, N$ & 176 & 134 \\
\hline Patients with score $=0$ at week 24 & $144(81.8)$ & $76(56.7)$ \\
\hline$p$ value vs. adalimumab & $<0.001$ & \\
\hline \multicolumn{3}{|l|}{ Personal relationships } \\
\hline Patients with baseline score $=6, N$ & 68 & 44 \\
\hline Patients with score $=0$ at week 24 & $45(66.2)$ & $15(34.1)$ \\
\hline$p$ value vs. adalimumab & 0.073 & \\
\hline \multicolumn{3}{|l|}{ Treatment } \\
\hline Patients with baseline score $=3, N$ & 124 & 94 \\
\hline Patients with score $=0$ at week 24 & $89(71.8)$ & $54(57.4)$ \\
\hline$p$ value vs. adalimumab & 0.030 & \\
\hline
\end{tabular}

All values are $n(\%)$ unless noted otherwise

Domains with one question have a total maximum score (most severe) of 3; domains with two questions have a total maximum score (most severe) of 6

measures, were associated with improved patient HRQoL. The proportion of guselkumab-treated patients who achieved a DLQI of 0/1 increased with PASI improvement. The same trend was observed for IGA responses. Likewise, the correlation between greater skin clearance and better HRQoL was also observed with adalimumab treatment. These findings support the relationship between skin clearance and better HRQoL [25, 35] and further validate the increasing emphasis on patient-reported outcomes in evaluating clinical responses to treatment. It is notable that, among patients who achieved either PASI 100 or IGA 0 , higher proportions of guselkumab-treated patients than adalimumab-treated patients achieved a DLQI of $0 / 1$, suggesting that factors other than complete skin clearance may also drive impact on HRQoL and that patient-reported outcomes may capture additional treatment benefits beyond PASI or IGA. Further investigation and validation is required to better understand this finding.

The major limitations of the study include a limited follow-up of 24 weeks, the restricted experience to date with the PSSD, and the limitations of the DLQI. While the DLQI is a validated instrument, it is not psoriasis-specific and does not assess the impact of signs and symptoms of psoriasis on HRQoL. The DLQI has limitations in some other aspects of psychometric performance, particularly unidimensionality and item bias [36-38], as well as the biases related to "not relevant" responses and issues with content validity [39-41]. As a result, multiple psoriasis-specific patientreported outcome instruments have been developed and validated in recent years to assess newer biologics [10-12]. Strengths of the study include the substantial database from the pooled study, the comparison against adalimumab, and the use of the validated, psoriasis-specific PSSD. Notably, greater improvements in patient-reported outcomes were demonstrated with guselkumab treatment compared with adalimumab treatment in the VOYAGE 1 and VOYAGE 2 primary studies using the PSSD [16, 17], and in the analysis of pooled data in this report likely due in part to greater skin clearance with guselkumab. Further, we demonstrated that improvements in HRQoL, as measured by achieving a DLQI 0/1, correlated with improvements in patient-reported symptoms and signs as assessed by achieving PSSD scores of 0 .

\section{Conclusion}

Pooled data from the phase III VOYAGE 1 and VOYAGE 2 studies demonstrated that guselkumab was superior to adalimumab in improving HRQoL through 24 weeks of treatment as assessed by multiple DLQI endpoints and patient-assessed symptoms and signs evaluated by the PSSD. The comparative data between guselkumab and adalimumab in a substantial cohort are novel and useful in treatment decisions, particularly with inclusion of the PSSD, which is a psoriasis-specific validated patient assessment. In addition, a distinct association between 
Table 5 Proportion of patients with Dermatology Life Quality Index (DLQI) scores of 0/1 at week $24^{\text {a }}$ by Psoriasis Area and Severity Index responses, Investigator's Global Assessment scores, and Patient Symptoms and Signs Diary scores

\begin{tabular}{|c|c|c|c|c|}
\hline & Placebo $\rightarrow$ guselkumab & Guselkumab & Adalimumab & Total \\
\hline Patients randomized at week $0, N$ & 422 & 825 & 582 & 1829 \\
\hline Patients with baseline DLQI score $>1, N$ & 390 & 811 & 565 & 1766 \\
\hline \multicolumn{5}{|l|}{ Psoriasis Area and Severity Index (PASI) } \\
\hline Patients achieving PASI 100, $N$ & 73 & 360 & 146 & 579 \\
\hline Achieved DLQI 0/1 & $52(71.2)$ & $286(79.4)$ & $96(65.8)$ & $434(75.0)$ \\
\hline Patients achieving PASI 90 to $<$ PASI $100, N$ & 112 & 263 & 158 & 533 \\
\hline Achieved DLQI 0/1 & $46(41.1)$ & $145(55.1)$ & $85(53.8)$ & $276(51.8)$ \\
\hline Patients achieving PASI 75 to $<$ PASI $90, N$ & 124 & 105 & 101 & 330 \\
\hline Achieved DLQI 0/1 & $47(37.9)$ & $40(38.1)$ & $36(35.6)$ & $123(37.3)$ \\
\hline Patients achieving $<$ PASI 75, $N$ & 81 & 83 & 160 & 324 \\
\hline Achieved DLQI 0/1 & $16(19.8)$ & $7(8.4)$ & $10(6.3)$ & $33(10.2)$ \\
\hline \multicolumn{5}{|l|}{ Investigator's Global Assessment (IGA) } \\
\hline Patients achieving IGA of cleared (0), $N$ & 107 & 422 & 171 & 700 \\
\hline Achieved DLQI 0/1 & $69(64.5)$ & $320(75.8)$ & $114(66.7)$ & $503(71.9)$ \\
\hline Patients achieving IGA of minimal (1), $N$ & 192 & 255 & 184 & 631 \\
\hline Achieved DLQI 0/1 & $69(35.9)$ & $127(49.8)$ & $80(43.5)$ & $276(43.7)$ \\
\hline Patients achieving IGA $\geq 2, N$ & 91 & 134 & 210 & 435 \\
\hline Achieved DLQI 0/1 & $23(25.3)$ & $31(23.1)$ & $33(15.7)$ & $87(20.0)$ \\
\hline \multicolumn{5}{|l|}{ Patient Symptoms and Signs Diary (PSSD) } \\
\hline PSSD symptom score $=0$ and baseline $>0, N$ & 39 & 232 & 102 & 373 \\
\hline Achieved DLQI 0/1 & $31(79.5)$ & $219(94.4)$ & $85(83.3)$ & $335(89.8)$ \\
\hline PSSD symptom score $>0$ and baseline $>0, N$ & 268 & 421 & 364 & 1053 \\
\hline Achieved DLQI of $0 / 1$ & $86(32.1)$ & $162(38.5)$ & $97(26.6)$ & $345(32.8)$ \\
\hline PSSD sign score $=0$ and baseline $>0, N$ & 25 & 186 & 72 & 283 \\
\hline Achieved DLQI 0/1 & $21(84.0)$ & $176(94.6)$ & $64(88.9)$ & $261(92.2)$ \\
\hline PSSD sign score $>0$ and baseline $>0, N$ & 282 & 468 & 396 & 1146 \\
\hline Achieved DLQI 0/1 & $96(34.0)$ & $206(44.0)$ & $120(30.3)$ & $422(36.8)$ \\
\hline
\end{tabular}

All values are $n(\%)$ unless noted otherwise

${ }^{a}$ In patients randomized to placebo, after week 16 the data includes only those patients who crossed-over to guselkumab at or after week 16

HRQoL improvement and greater skin clearance (PASI and IGA) was identified, with a trend for guselkumab to provide greater patient-perceived benefit than adalimumab in those with clear skin. This suggestion that patient-reported outcomes capture greater benefit than standard physician measurement of psoriasis is intriguing and requires further investigation. Finally, the correlation between the well-established DLQI and the novel PSSD is reassuring.

Acknowledgements The authors thank Kristin Ruley Sharples, $\mathrm{PhD}$, of Janssen Scientific Affairs, LLC (Spring House, PA, USA) and Cynthia Guzzo, MD, of HireGenics (Duluth, GA, USA) for their writing and editorial support in the preparation of this manuscript.

\section{Compliance with Ethical Standards}

Funding This study was funded by Janssen Research \& Development, LLC.
Conflict of interest A Armstrong has received grant funding from AbbVie, Eli Lilly, and Janssen, and consulting fees/honorarium from AbbVie, Janssen, Eli Lilly, Leo Pharma, Modernizing Medicine, Novartis, Ortho Dermatologics, Regeneron, Sanofi, and Science 37. K. Reich has served as an advisor and/or paid speaker for and/or participated in clinical trials sponsored by AbbVie, Affibody, Almirall, Amgen, Biogen, Boenhringer Ingelheim, Celgene, Covagen, Eli Lilly, Forward Pharma, Frensenius Medical Care, GlaxoSmtihKline, Janssen, Janssen-Cilag, Kyowa Kirin, Leo Pharma, Medac, Merck Sharp \& Dohme, Novartis, Miltenyi Biotec, Ocean Pharma, Pfizer, Regeneron, Samsung Bioepis, Sanofi, Takeda, UCB, Valeant, and Xenoport. P. Foley has received research grants from Abbott/AbbVie, Amgen, Celgene, Eli Lilly, Janssen, Novartis, Shering-Plough/MSD, and Wyeth/Pfizer; served as an advisory board member for Abbott/AbbVie, Amgen, Biogen Idec, Celgene, Eli Lilly, Shering-Plough/MSD, Galderma, Janssen, Leo Pharma/Peplin, Novartis, Sanofi Genzyme, Sun Pharma, UCB, and Wyeth/Pfizer; received travel support for investigators' meetings for clinical trials as the Principal Investigator; and received speaker bureau fees from $3 \mathrm{M} / \mathrm{iNova} /$ Valeant, Abbott/ AbbVie, Biogen Idec, Celgene, Eli Lilly, Galderma, GSK/Stiefel, Janssen, Leo Pharma/Peplin, Novartis, Shering-Plough/MSD, and Wyeth/ Pfizer. K. Papp has served as a consultant and/or scientific advisor and/or 
investigator and/or scientific officer and/or speaker for AbbVie, Akros, Allergan, Amgen, Anacor, Arcutis, Astrellas, AstraZeneca, Baxalta, Baxter, Boehringer Ingelheim, BMS, CanFite, Celgene, Coherus, Demira, Dow Pharma, Eli Lilly, Forward Pharam, Galderma, Genentech, GlaxoSmithKline, Janssen, Kyowa Hakko Kirin, Leo Pharma, Medimmune, Meiji Seiki Pharma, Merck Sharp \& Dohme, MerckSerono, Mitsubishi Pharma, Novartis, Pfizer, Regeneron, Roche, Sanofi-Aventis/Genzyme, Takeda, UCB, and Valeant. C. Han, M. Song, Y.-K. Shen, and Y. You are employees of Janssen Research \& Development, LLC. and own stock in Johnson \& Johnson, of which Janssen is a subsidiary.

Ethical approval/informed consent All procedures performed in studies involving human participants were in accordance with the ethical standards of the institutional and/or national research committee and with the 1964 Declaration of Helsinki and its later amendments or comparable ethical standards. All patients provided written informed consent prior to any study-related procedures.

Open Access This article is distributed under the terms of the Creative Commons Attribution-NonCommercial 4.0 International License (http://creativecommons.org/licenses/by-nc/4.0/), which permits any noncommercial use, distribution, and reproduction in any medium, provided you give appropriate credit to the original author(s) and the source, provide a link to the Creative Commons license, and indicate if changes were made.

\section{References}

1. Dalgard FJ, Gieler U, Tomas-Aragones L, et al. The psychological burden of skin diseases: a cross-sectional multicenter study among dermatological out-patients in 13 European countries. J Invest Dermatol. 2015;135:984-91.

2. Bundy C, Borthwick M, McAteer H, et al. Psoriasis: snapshots of the unspoken: using novel methods to explore patients' personal models of psoriasis and the impact on well-being. Br J Dermatol. 2014;171:825-31.

3. Kimball AB, Jacobson C, Weiss S, Vreeland MG, Wu Y. The psychosocial burden of psoriasis. Am J Clin Dermatol. 2005;6:383-92.

4. Finlay AY, Khan GK. Dermatology Life Quality Index (DLQI)-a simple practical measure for routine clinical use. Clin Exp Dermatol. 1994;19:210-6.

5. Finlay AY, Khan GK. Dermatology Life Quality Index. http:// www.bad.org.uk. Accessed Apr 1992.

6. Jaeschke R, Singer J, Guyatt GH. Measurement of health status. Ascertaining the minimal clinically important difference. Control Clin Trials. 1989;10:407-15.

7. Shikiar R, Willian MK, Okun MM, Thompson CS, Revicki DA. The validity and responsiveness of three quality of life measures in the assessment of psoriasis patients: results of a phase II study. Health Qual Life Outcomes. 2006;4:71.

8. Strand V, Fiorentino D, Hu C, Day RM, Stevens RM, Papp KA. Improvements in patient-reported outcomes with apremilast, an oral phosphodiesterase 4 inhibitor, in the treatment of moderate to severe psoriasis: results from a phase IIb randomized, controlled study. Health Qual Life Outcomes. 2013;11:82.

9. Basra MK, Salek MS, Camilleri L, Sturkey R, Finlay AY. Determining the minimal clinically important difference and responsiveness of the Dermatology Life Quality Index (DLQI): further data. Dermatology. 2015;230:27-33.
10. Armstrong A, Puig L, Langley R, Kim E, Gwaltney CJ, Strober BE. Validation of psychometric properties and development of response criteria for the Psoriasis Symptoms and Signs Diary (PSSD): results from a phase III clinical trial. J Dermatolog Treat. 2018;11:1-31. https://doi.org/10.1080/09546634.2017.1364694 (Epub ahead of print).

11. Lebwohl M, Swensen AR, Nyirady J, Kim E, Gwaltney CJ, Strober BE. The Psoriasis Symptom Diary: development and content validity of a novel patient-reported outcome instrument. Int J Dermatol. 2014;53:714-22.

12. Martin ML, McCarrier KP, Chiou CF, et al. Early development and qualitative evidence of content validity for the Psoriasis Symptom Inventory (PSI), a patient-reported outcome measure of psoriasis symptom severity. J Dermatolog Treat. 2013;24:255-60.

13. Mathias SD, Feldman SR, Crosby RD, Colwell HH, McQuarrie K, Han C. Measurement properties of a patient-reported outcome measure assessing psoriasis severity: the psoriasis symptoms and signs diary. J Dermatolog Treat. 2016;27:322-7.

14. Strober B, Papp KA, Lebwohl M, et al. Clinical meaningfulness of complete skin clearance in psoriasis. J Am Acad Dermatol. 2016;75(77-82):e7.

15. Blome C, Gosau R, Radtke MA, et al. Patient-relevant treatment goals in psoriasis. Arch Dermatol Res. 2016;308:69-78.

16. Blauvelt A, Papp KA, Griffiths CEM, et al. Efficacy and safety of guselkumab, an anti-interleukin-23 monoclonal antibody, compared with adalimumab for the continuous treatment of patients with moderate to severe psoriasis: results from the phase III, double-blinded, placebo- and active comparator-controlled VOYAGE 1 trial. J Am Acad Dermatol. 2017;76:405-17.

17. Reich K, Armstrong AW, Foley P, et al. Efficacy and safety of guselkumab, an anti-interleukin-23 monoclonal antibody, compared with adalimumab for the treatment of patients with moderate to severe psoriasis with randomized withdrawal and retreatment: results from the phase III, double-blind, placebo- and active comparator-controlled VOYAGE 2 trial. J Am Acad Dermatol. 2017;76:418-31.

18. Sofen H, Smith S, Matheson RT, et al. Guselkumab (an IL23-specific $\mathrm{mAb}$ ) demonstrates clinical and molecular response in patients with moderate-to-severe psoriasis. J Allergy Clin Immunol. 2014;133:1032-40.

19. Langley RG, Elewski BE, Lebwohl M, et al., ERASURE Study Group, FIXTURE Study Group. Secukinumab in plaque psoriasis-results of two phase 3 trials. N Engl J Med. 2014;371:326-38.

20. Reich K, Papp KA, Blauvelt A, et al. Tildrakizumab versus placebo or etanercept for chronic plaque psoriasis (reSURFACE 1 and reSURFACE 2): results from two randomised controlled, phase 3 trials. Lancet. 2017;390:276-88.

21. Griffiths CEM, Reich K, Lebwohl M, et al.; UNCOVER-2 and UNCOVER-3 investigators. Comparison of ixekizumab with etanercept or placebo in moderate-to-severe psoriasis (UNCOVER-2 and UNCOVER-3): results from two phase 3 randomised trials. Lancet. 2015;386:541-51.

22. Krueger JG, Ferris LK, Menter A, et al. Anti-IL-23A mAb BI 655066 for treatment of moderate-to-severe psoriasis: safety, efficacy, pharmacokinetics, and biomarker results of a singlerising-dose, randomized, double-blind, placebo-controlled trial. J Allergy Clin Immunol. 2015;136(116-24):e7.

23. Puig L, Thom H, Mollon P, Tian H, Ramakrishna GS. Clear or almost clear skin improves the quality of life in patients with moderate-to-severe psoriasis: a systematic review and meta-analysis. J Eur Acad Dermatol Venereol. 2017;31:213-20.

24. Mattei PL, Corey KC, Kimball AB. Psoriasis Area Severity Index (PASI) and the Dermatology Life Quality Index (DLQI): the correlation between disease severity and psychological burden in patients treated with biological therapies. J Eur Acad Dermatol Venereol. 2014;28:333-7. 
25. Viswanathan HN, Chau D, Milmont CE, et al. Total skin clearance results in improvements in health-related quality of life and reduced symptom severity among patients with moderate to severe psoriasis. J Dermatolog Treat. 2015;26:235-9.

26. Silva MFP, Parise-Fortes MR, Miot LBD, Marques SA. Psoriasis: correlation between severity index (PASI) and quality of life index (DLQI) in patients assessed before and after systemic treatment. An Bras Dermatol. 2013;88:760-3.

27. Abrouk M, Nakamura M, Zhu TH, Farahnik B, Koo J, Bhutani T. The impact of PASI 75 and PASI 90 on quality of life in moderate to severe psoriasis patients. J Dermatolog Treat. 2017;28:488-91.

28. Herédi E, Rencz F, Balogh O, et al. Exploring the relationship between EQ-5D, DLQI and PASI, and mapping EQ-5D utilities: a cross-sectional study in psoriasis from Hungary. Eur J Health Econ. 2014;15(Suppl 1):S111-9.

29. Norlin JM, Steen Carlsson K, Persson U, Schmitt-Egenolf M. Analysis of three outcome measures in moderate to severe psoriasis: a registry-based study of 2450 patients. Br J Dermatol. 2012;166:797-802.

30. Fredriksson T, Pettersson U. Severe psoriasis-oral therapy with a new retinoid. Dermatologica. 1978;157:238-44.

31. Barra L, Pope JE, Payne M. Real-world anti-tumor necrosis factor treatment in rheumatoid arthritis, psoriatic arthritis, and ankylosing spondylitis: cost-effectiveness based on number needed to treat to improve health assessment questionnaire. J Rheumatol. 2009;36:1421-8.

32. Reich K, Pinter A, Lacour JP, et al.; IXORA-S investigators. Comparison of ixekizumab with ustekinumab in moderate-to-severe psoriasis: 24-week results from IXORA-S, a phase III study. Br J Dermatol. 2017; 177:1014-23.

33. Puig L, Augustin M, Blauvelt A, et al. Effect of secukinumab on quality of life and psoriasis-related symptoms: a comparative analysis versus ustekinumab from the CLEAR 52-week study. J Am Acad Dermatol. 2018;78:741-8.

34. Blauvelt A, Reich K, Tsai TF, et al. Secukinumab is superior to ustekinumab in clearing skin of subjects with moderate-to-severe plaque psoriasis up to 1 year: results from the CLEAR study. J Am Acad Dermatol. 2017;76(60-9):e9.

35. Reich K, Griffiths CEM. The relationship between quality of life and skin clearance in moderate-to-severe psoriasis: lessons learnt from clinical trials with infliximab. Arch Dermatol Res. 2008;300:537-44.

36. Bronsard V, Paul C, Prey S, et al. What are the best outcome measures for assessing quality of life in plaque type psoriasis? A systematic review of the literature. J Eur Acad Dermatol Venereol. 2010;24(Suppl 2):17-22.

37. Nijsten T, Meads DM, McKenna SP. Dimensionality of the dermatology life quality index (DLQI): a commentary. Acta Derm Venereol. 2006;86:284-5.

38. Twiss J, Meads DM, Preston EP, Crawford SR, McKenna SP. Can we rely on the Dermatology Life Quality Index as a measure of the impact of psoriasis or atopic dermatitis? J Invest Dermatol. 2012;132:76-84.

39. Rencz F, Poór AK, Péntek M, et al. A detailed analysis of 'not relevant' responses on the DLQI in psoriasis: potential biases in treatment decisions. J Eur Acad Dermatol Venereol. 2018;32:783-90.

40. Poór AK, Brodszky V, Péntek M, et al. Is the DLQI appropriate for medical decision-making in psoriasis patients? Arch Dermatol Res. 2018;310:47-55.

41. Rencz F, Gulácsi L, Péntek M, et al. Proposal of a new scoring formula for the DLQI in psoriasis. Br J Dermatol. 2018. https:// doi.org/10.1111/bjd.16927 (Epub ahead of print). 